\title{
Application of an acellular dermal matrix to a rabbit model of oral mucosal defects
}

\author{
XIANGLIANG XU, NIANHUI CUI and ENBO WANG \\ Department of Oral and Maxillofacial Surgery, \\ Peking University School and Hospital of Stomatology, Beijing 100081, P.R. China
}

Received May 11,2017; Accepted October 6, 2017

DOI: $10.3892 /$ etm.2018.5705

\begin{abstract}
Acellular dermal matrices (ADMs) are increasingly used for the restoration of soft-tissue defects of the oral cavity due to their ability to facilitate faster healing and reduce scar formation without rejection. However, few studies have focused on the histopathology and biological mechanisms involved in their use. The aim of the present study was to observe tissue growth, histopathologic changes and altered biomolecular signatures that occur during the repair of oral defects in rabbit models over time, either with or without the employment of ADM. Animals were sacrificed 1, 2 and 4 weeks following surgery and histological changes were evaluated using hematoxylin and eosin staining. Reverse transcription-polymerase chain reaction and western blot analysis were used to determine changes in the expression of vascular endothelial growth factor (VEGF) and glucose transporter 1 (GLUT1). It was demonstrated that wounds treated with ADM exhibited a weak inflammatory reaction and faster epithelialization and revascularization compared with untreated wounds. This may have been caused by the elevated levels of VEGF and GLUT1 protein detected in the ADM-treated defects. Thus, treating wounds of the oral mucosa with an ADM improves pathological responses compared with those with an untreated wound. The current study demonstrates the underlying mechanisms by which ADM promotes wound healing in defects of the oral mucosa and the results provide further evidence for the use of ADM in clinical settings for the repair of mucosal defects.
\end{abstract}

Correspondence to: Dr Enbo Wang, Department of Oral and Maxillofacial Surgery, Peking University School and Hospital of Stomatology, 22 Zhongguancun South Street, Haidian, Beijing 100081, P.R. China

E-mail: liang201704@126.com

Abbreviations: ADM, acellular dermal matrix; VEGF, vascular endothelial growth factor; GLUT1, glucose transporter 1

Key words: acellular dermal matrix, oral mucosa defects, rabbit models, repair, vascular endothelial growth factor, glucose transporter 1

\section{Introduction}

Oral mucosal lesions, facial trauma and management of tumors of the oral cavity often cause oral mucosal defects. Repair of the oral mucosa requires tissue engineering or transplantation as large defects cannot be directly sutured (1). Split-thickness skin grafts are considered the 'gold standard' for the treatment of skin wounds (2). However, the small size of donor oral tissue, limited graft survival due to the full thickness nature of mucosal grafts and contracture/scarring at the donor site have all limited the use of mucosal grafts for the treatment of oral wounds (3). In addition, due to the differences between skin and oral mucosa, the transplanted skin grafts are poorly assimilated even years after transplantation (4). Different approaches to the reconstruction of oral mucosa have been employed in attempts to find a solution to this issue. These include oral epithelial cell sheets, guided tissue replacement and vestibuloplasty (5). However, identifying an acceptable source for transplantations or autologous grafts is challenging (6).

Acellular dermal matrix (ADM), processed from allogeneic human skin, has been proposed as an alternative approach for the treatment of oral mucosal wounds (7). Following removal of the keratin-containing epidermis, the dermal layer is processed to remove all DNA without disrupting the collagen matrix. This preparation allows for host cell infiltration, neovascularization and epithelialization without rejection (8). Rhee et al (9) used commercially available ADM as a substitute for autologous, split-thickness skin grafts for the resurfacing of intraoral defects in 29 patients. The overall success rate was $90 \%$, with complete epithelialization within 4 weeks noted during clinical evaluation. The clinical application of ADM has yielded promising results and high success rates. However, the changes in histopathology and molecular biology that occur during the epithelialization process following transplantation into the oral cavity remain unclear. To the best of our knowledge, few studies have investigated the functional properties of ADM in oral mucosal repair in animal models. Thus, the present study was designed to assess the repair of oral mucosal defects in rabbits over time using ADM and to investigate the potential biological mechanisms underlying its efficacy in order to further support and inform the use of this strategy in clinical settings. 


\section{Materials and methods}

Animals. In total, 30 healthy adult (25-28 weeks) male New Zealand white rabbits weighing 4.0-4.5 kg were used in the present study (Peking University Laboratory Animal Center, Beijing, China). All rabbits were acclimated in the experimental animal laboratory of Peking University Hospital of Stomatology for 1 week prior to the experiment. Rabbits were maintained on a $14 \mathrm{~h}$ light $/ 8 \mathrm{~h}$ dark cycle at $23 \pm 2^{\circ} \mathrm{C}$ and $60-70 \%$ air humidity with free access to food and water. Experimental procedures were performed in accordance with the Guidelines for Animal Experimentation of Peking University with the approval of the Animal Care and Use Committee at Peking University School and Hospital of Stomatology.

Study design. Animals were assigned to one of two groups. The rabbits in the ADM group $(n=15)$ underwent oral mucosal defect surgery and were treated with an ADM covering and iodoform gauze. The rabbits in the control group $(n=15)$ were treated with only iodoform gauze following oral mucosal defect surgery. At 1,2, and 4 weeks after the surgery, 5 animals per group were sacrificed in order to perform histological investigations. The study design is presented in Fig. 1.

Surgical procedures. Rabbits were anesthetized with intravenous pentobarbital sodium $(30 \mathrm{mg} / \mathrm{kg}$ ). Following disinfection with $1 \%$ iodine tincture and local anesthesia with $1 \%$ lidocaine, the palate was fully exposed (Fig. 2A). Palatal mucosa was carefully separated from the underlying submucosa backwards from the fourth palatal rugae and was then excised, ensuring that the bilateral incision did not exceed the outer boundary of the palatal mucosa. The deficient area was $\sim 1 \mathrm{x} 1 \mathrm{~cm}^{2}$ (Fig. 2B). Adequate hemostasis was then ensured in the mucosa-deficient region (Fig. 2C). Subsequently, for the ADM group, ADM (1x1 cm; Beijing Jayyalife Biological Technology Co., Ltd., Beijing, China) was hydrated with two saline washes according to the manufacturer's protocol (Fig. 2D) and was then cut to the same size as the wound surface. The ADM was then embedded in the defect region and attached as a covering tissue with a simple interrupted suture with 3-0 stitches (Fig. 2E). Wounds were then covered with iodoform gauze and wrapped together with ADM (Fig. 2F). In the control group, the wound surface was covered with iodoform gauze only. Surgery was performed under sterile conditions. Following surgery, all rabbits were fasted and deprived of water for $12 \mathrm{~h}$ and then provided only soft food for 1 week. Following removal of the iodoform gauze under intravenous anesthesia with $30 \mathrm{mg} / \mathrm{kg} 3 \%$ pentobarbital sodium via the ear vein, rabbits were returned to a diet of normal pellets and water.

Histological procedures. All rabbits were sacrificed with an overdose of pentobarbital sodium $(>120 \mathrm{mg} / \mathrm{kg})$ through the ear vein. The front jaw was removed and the soft tissue of the palate, together with the corresponding deep bone tissue were immediately collected and fixed in $10 \%$ buffered formalin for 2 days at room temperature. The periosteum of the palate was then bluntly separated and the wound region was obtained, together with $2-3 \mathrm{~cm}$ of normal palatal mucosa around the operative region. Following routine procedures, tissues were embedded in paraffin and $10-\mu \mathrm{m}$ sections were prepared for histological evaluation. Hematoxylin and eosin (H\&E) staining was performed according to a previously published protocol (10) to evaluate the morphology of the palate mucosa under a light microscope.

Reverse transcription-quantitative polymerase chain reaction $(R T-q P C R)$. Total RNA was extracted from tissue sections that had been stored in liquid nitrogen using TRIzol reagent (Invitrogen; Thermo Fisher Scientific, Inc., Waltham, MA, USA) according to the manufacturer's protocol. cDNA was then synthesized and quantified using the PrimeScript RT reagent kit (Takara Biotechnology Co., Ltd., Dalian, China) on an ABI 7300 System (Applied Biosystems; Thermo Fisher Scientific, Inc.). The reaction was performed at $16^{\circ} \mathrm{C}$ for $30 \mathrm{~min} ; 42^{\circ} \mathrm{C}$ for $30 \mathrm{~min}$ and $85^{\circ} \mathrm{C}$ for $5 \mathrm{~min}$. qPCR was carried out using a Power SYBR Green PCR master mix (Applied Biosystems; Thermo Fisher Scientific, Inc.). Primers sequences were as follows: GLUT1, forward, 5'-CAGTTC GGCTATAACACTGGTG-3' and reverse, 5'-GCCCCCGAC AGAGAAGATG-3'; VEGF, forward, 5'-TGCCCACTGAGG AGTCCAAC-3' and reverse, 5'-TGGTTCCCGAAACGCTGA G-3' (Guangzhou RiboBio Co., Ltd., Guangzhou, China); and GAPDH, forward, 5'-GCACCGTCAAGGCTGAGAAC-3' and reverse, 5'-ATGGTGGTGAAGACGCCAGT-3'. PCR reactions were performed using the following conditions: $94^{\circ} \mathrm{C}$ for $1 \mathrm{~min}$ followed by 40 cycles of $94^{\circ} \mathrm{C}$ for $30 \mathrm{sec}, 60^{\circ} \mathrm{C}$ for $60 \mathrm{sec}$ and $72^{\circ} \mathrm{C}$ for $15 \mathrm{sec}$. Amplification of the target genes was normalized to GAPDH. All reactions were performed in triplicate. The $2^{-\Delta \Delta \mathrm{Cq}}$ method (11) was used to determine the relative expression of VEGF and GLUT1.

Western blot analysis. Total protein was extracted from the epithelium of the wound tissue using PRO-PREP (Intron Biotechnology, Inc., Seongnam, Korea), according to the manufacturer's protocol and the BCA protein determination method was used. Equal amounts of protein (30 $\mu \mathrm{g}$ per lane) were separated by $12 \%$ SDS-PAGE, transferred onto PVDF membranes (EMD Millipore, Billerica, MA, USA) and blocked with $10 \%$ bovine serum albumin (Sigma-Aldrich; Merck KGaA, Darmstadt, Germany) at room temperature for $2 \mathrm{~h}$. Membranes were then incubated with antibodies specific to GLUT1 (1:1,000; cat no. NB110-39113; Novus Biologicals Canada ULC, Oakville, ON, Canada), vascular endothelial growth factor (VEGF) (1:500; cat no. MAB293R; R\&D Systems, Inc., Minneapolis, MN, USA) and GAPDH (1:2,000; cat no. GTX100118; GeneTex, Inc., Irvine, CA, USA) overnight at $4^{\circ} \mathrm{C}$, followed by incubation with anti-rabbit Immunoglobulin $\mathrm{G}$ horseradish peroxidase-conjugated antibodies (1:5,000; cat no. 7071; Cell Signaling Technology, Inc., Danvers, MA, USA) for $2 \mathrm{~h}$ at room temperature. Immunoreactive bands were detected using an ECL detection system (cat no. 6883, Cell Signaling Technology, Inc.) and analyzed with Quantity One 4.0 software (Bio-Rad Laboratories, Inc., Hercules, CA, USA).

Statistical analysis. Data are expressed as mean \pm standard deviation. Group comparisons were calculated using SPSS 19.0 software (IBM Corp., Armonk, NY, USA) and unpaired, two-tailed Student's t-tests. $\mathrm{P}<0.05$ was considered to indicate a statistically significant difference. 


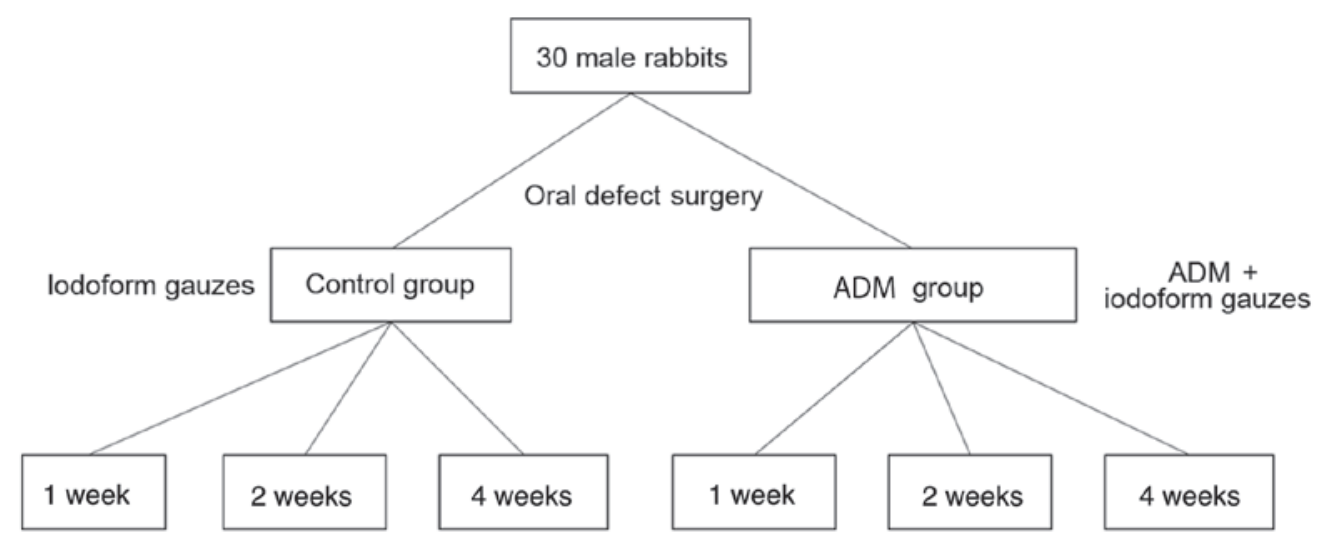

Figure 1. Study design. ADM, acellular dermal matrix.

A

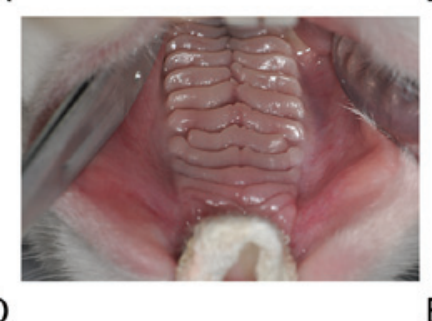

D

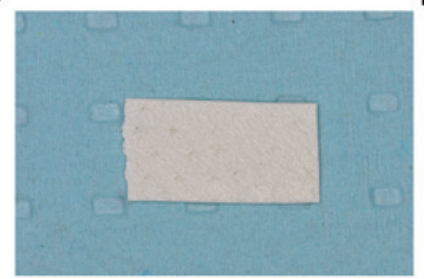

B

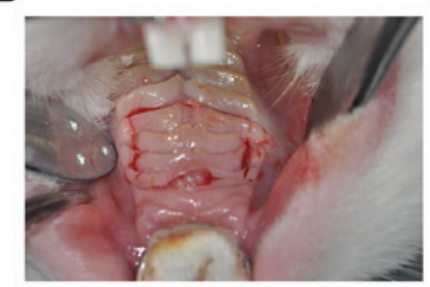

$E$

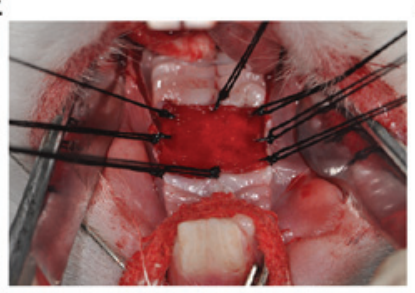

C

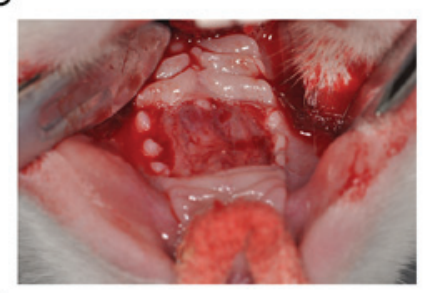

$\mathrm{F}$

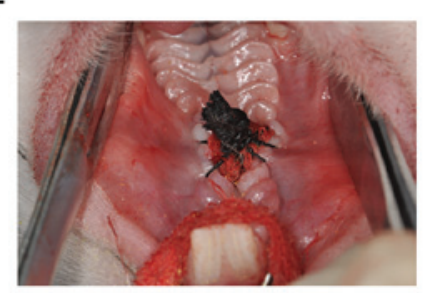

Figure 2. Representative images of the surgical procedure. (A) Fully exposed palate following anesthesia. (B) Manufacture of the border of artificial damage on the palatal mucosa. (C) Extraction of the palatal rugae to form defects of the oral mucosa. (D) ADM cut to the size of the defect. (E) ADM embedded in each defect via simple interrupted suture. (F) Wound wrapped with iodoform gauze. ADM, acellular dermal matrix.

\section{Results}

Clinical observation. No hematomas or inflammation were observed in rabbits following surgery. At 1 week following surgery, superficial ulcers and some necrotic tissue were observed in the operative site of animals in the ADM group. The boundaries between the operative region and normal mucosal were unclear, and granulation tissue continuous with the connective tissue of the oral mucosa was observed (Fig. 3A). By comparison, rabbits in the control group displayed deeper ulcers and more necrotic tissue, with new granulation tissue filling the surgical wounds (Fig. 3B). At 2 weeks following surgery, the ulcers were significantly reduced in the ADM group and the mucosa had begun to form with a tenacious texture. However, the palatal rugae remained unclear (Fig. 3C). By contrast, despite the boundaries between the operative region and normal mucosal tissue becoming less clearly defined, ulcers remained relatively large and dispersed necrotic tissue remained present in the control group (Fig. 3D). At 4 weeks following surgery, all animals from the ADM and control groups had recovered and palatal rugae were resurgent, with no ulcers or necrosis in the operative area (Fig. 3E and F).
Histopathological analysis. At 1 week following surgery in the ADM group, H\&E staining indicated a moderate degree of cellulose-like exudation on the surface of epithelial defects, suggesting that epithelialization was incomplete. In addition, the ADM collagen was filled with a large number of inflammatory and endothelial cells. Collagen fibers were arranged in fascicular clusters in the lower layer of the oral mucosa and the defect border had regularly arranged, neonatal epithelial tissue growing into the inflammatory site (Fig. 4A and B). Inflammatory cells were predominantly lymphocytes and granulocytes, with a lower proportion of macrophages. Early revascularization of the dermal equivalent was noted. However, in the control group, a larger amount of cellulose-like exudate was observed and the submucosa had fewer collagen fibers, which were randomly oriented (Fig. 4C). There was also an increased number of inflammatory cells compared with the ADM group. Epithelialization of the sutured wound progressed in the ADM group 2 weeks following surgery (Fig. 4D). However, keratinization was not complete and epithelial ridges were not present. Fibroblasts with large nuclei remained present in the submucosa, as well as a small number of inflammatory cells. Some vascularization was observed at the ADM graft sites. Histopathology of the wound area in the control group 

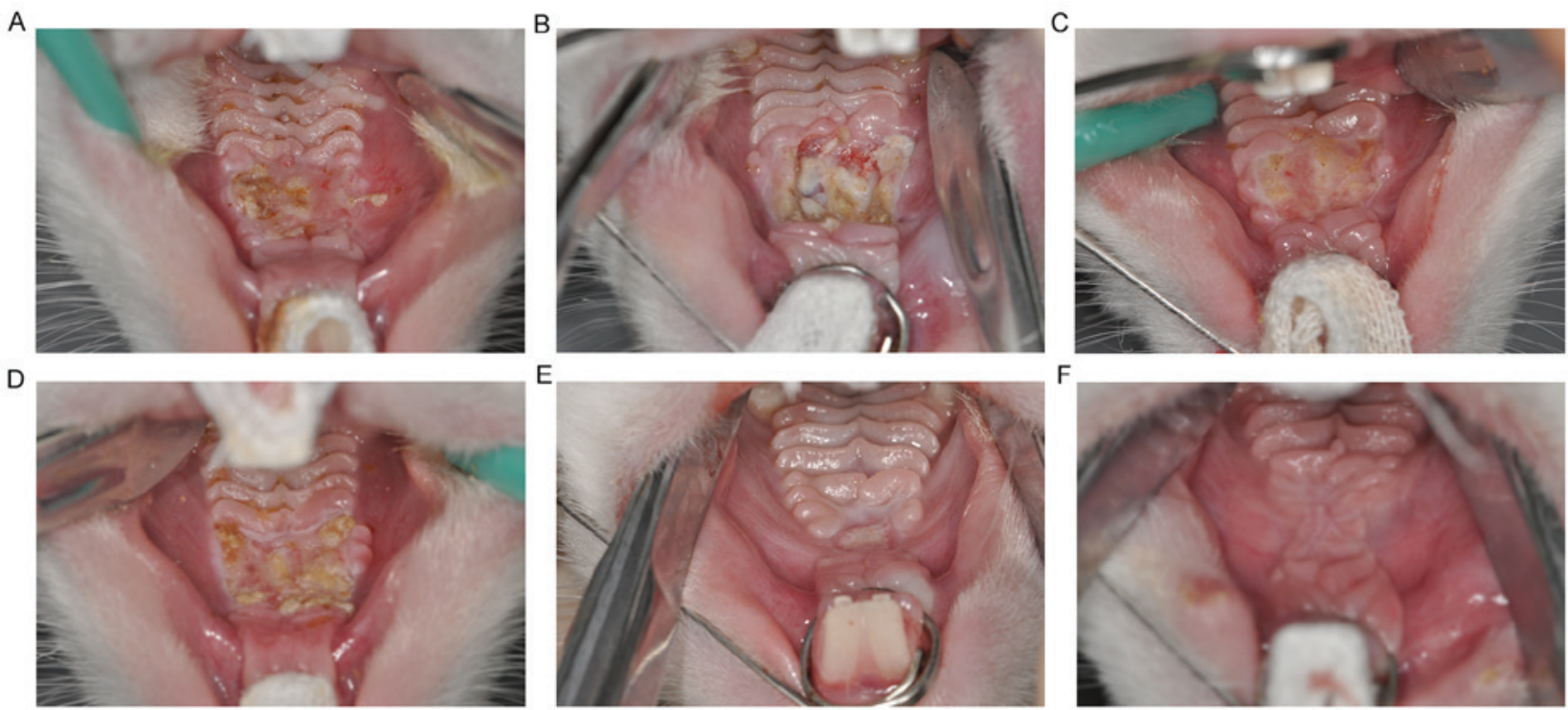

Figure 3. Gross appearance of wound healing following surgery. Wound healing at 1 week following surgery in the (A) ADM and (B) control groups. Wound healing at 2 weeks following surgery in the (C) ADM and (D) control groups. Wound healing at 4 weeks following surgery in the (E) ADM and (F) control groups. ADM, acellular dermal matrix.

A
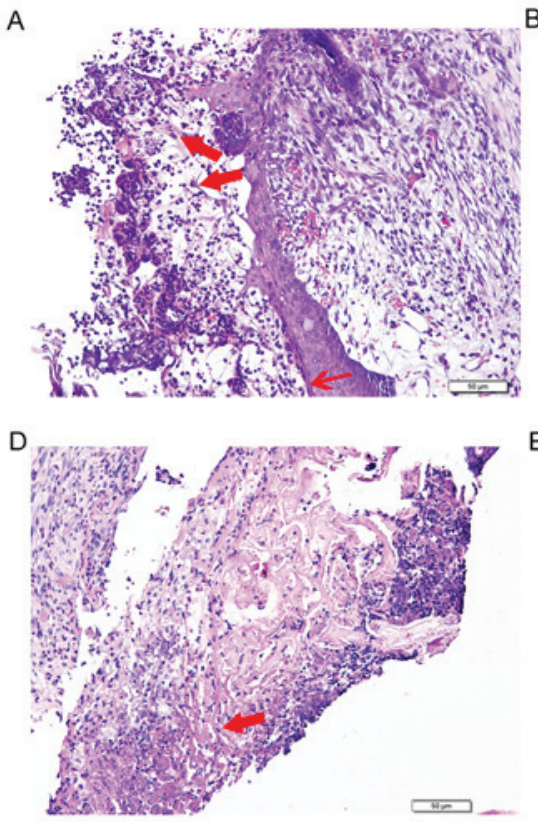

$B$
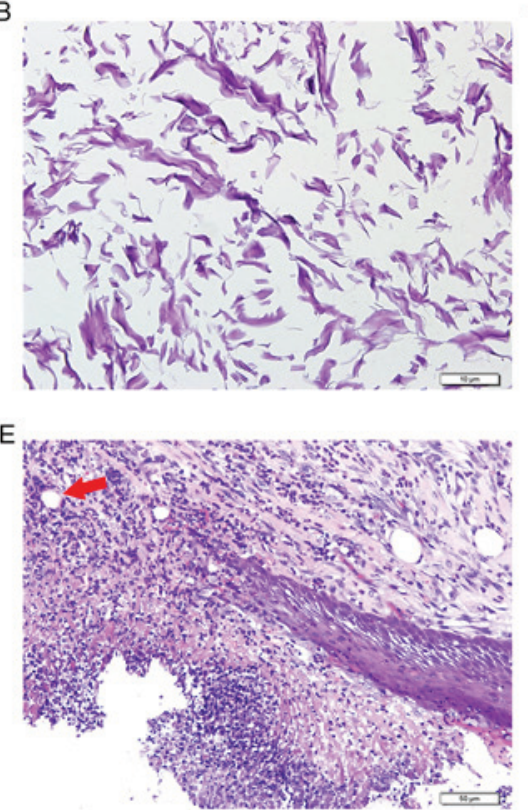

C

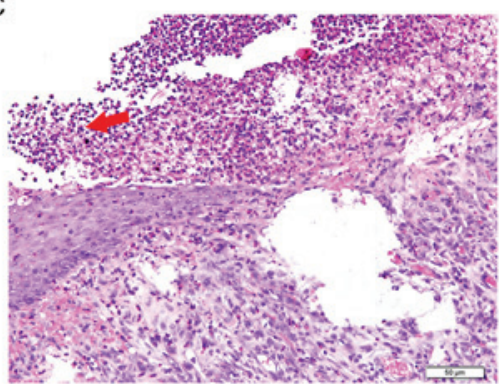

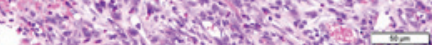

Figure 4. Histopathological analysis of wound healing progress via hematoxylin and eosin staining. (A) One week following surgery in the ADM group. Inflammatory cells (large arrow) were observed and collagen fibers were arranged in the lower layer of the oral mucosa (small arrow). Scale bar, $50 \mu \mathrm{m}$. (B) The ADM was infiltrated by inflammatory cells and endothelial cells 1 week following surgery. Scale bar, $10 \mu \mathrm{m}$. (C) Control group 1 week following surgery. Cellulose-like exudate was observed (arrow) with fewer collagen fibers. Scale bar, $50 \mu \mathrm{m}$. (D) ADM group 2 weeks following surgery. Epithelialization of the sutured wound progressed with parakeratosis (arrow). Scale bar, $50 \mu \mathrm{m}$. (E) Control group 2 weeks following surgery. Minimal vascularization was noted at the margin of the defects (arrow). Scale bar, $50 \mu \mathrm{m}$. ADM, acellular dermal matrix.

2 weeks following surgery was comparable to that seen in the ADM group 1 week following surgery (Fig. 4E). Cellulose-like exudate was present on the surface of the wound area and acute inflammatory cells that had infiltrated the collagen fibers were present in the submucosa. Minimal vascularization was noted at the margin of the defects. At 4 weeks following surgery, the defects were covered by well-organized, stratified squamous epithelium, and appeared similar to the normal surrounding mucosa in the control and ADM groups (data not shown).
Molecular biological investigation. The molecular biological alterations occurring during wound healing following the treatment with ADM were investigated. The difference in the expression of vascular endothelial growth factor (VEGF), which is a well-known angiogenic factor that is important for vascular development and maintenance during wound healing, between the control and ADM groups at 1 week post-surgery was not significant at mRNA and protein levels (Fig. 5A-C). However, the expression of VEGF was significantly increased 


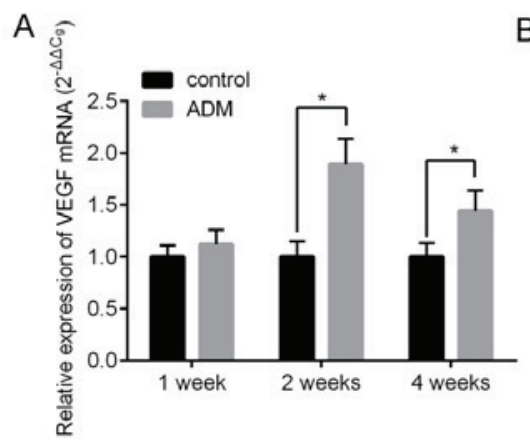

B

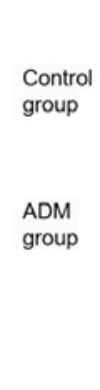

C

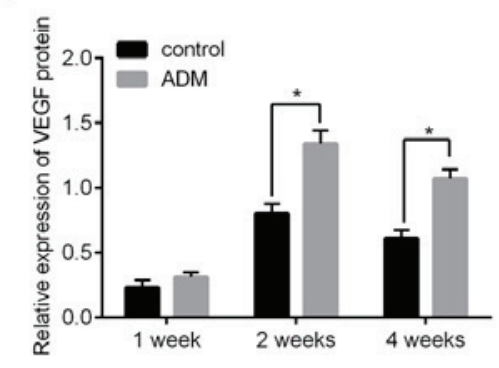

Figure 5. Expression of VEGF in the control and ADM groups. (A) Expression of VEGF mRNA determined using reverse transcription-quantitative polymerase chain reaction. (B) Western blot analysis of VEGF protein. (C) Quantification of western blot analysis. Data are expressed as mean \pm standard deviation of three repeated experiments. ${ }^{*} \mathrm{P}<0.05$. VEGF, vascular endothelial growth factor; ADM, acellular dermal matrix.
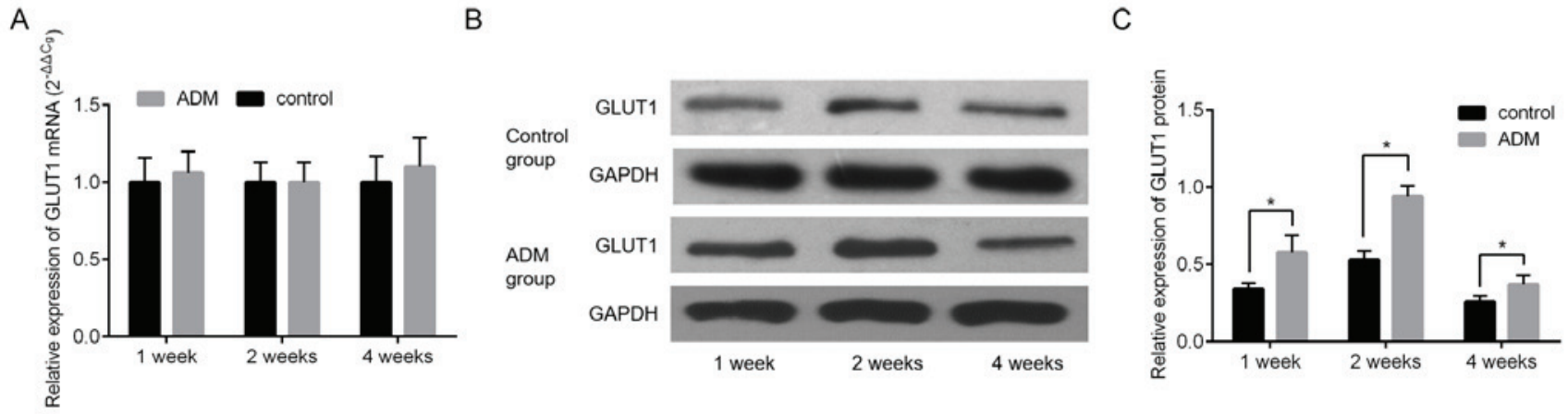

Figure 6. GLUT1 expression in oral mucosal defects of rabbits. (A) Expression of GLUT1 mRNA determined using reverse transcription-quantitative polymerase chain reaction. (B) Expression of GLUT1 protein determined using western blot analysis. (C) Quantification of western blot analysis. Data are expressed as mean \pm standard deviation of three repeated experiments. "P<0.05. GLUT1, glucose transporter 1; ADM, acellular dermal matrix.

in the ADM group compared with the control group at 2 and 4 weeks following surgery (Fig. 5A-C, P $<0.05$ ).

The expression of GLUT1 was measured using RT-qPCR and western blotting. The difference in the expression of GLUT1 mRNA between the control and ADM groups was not significant at all time points (Fig. 6A). However, GLUT1 protein expression was significantly increased in the ADM group compared with the control group at all time points (all $\mathrm{P}<0.05$, Fig. 6B and C).

\section{Discussion}

Wainwright (12) was the first to illustrate the use of an ADM processed from allogeneic human skin for the treatment of a full-thickness burn injury and since then a variety of reconstructive procedures utilizing ADM have emerged. These include supporting lymphangiogenesis (13), abdominal wall reconstructions (14) and postmastectomy reconstruction $(15,16)$. In the reconstruction of ridge deformities following tooth extraction in humans, ADM was novelly employed as a membrane associated with resorbable hydroxyapatite for bone regeneration, resulting in the preservation of ridge thickness and an increase in the width of keratinized tissue (17). When associated with an organic bovine bone matrix and P-15, a synthetic compound containing 15 amino acids of collagen type I, ADM exhibited the effective preservation of the alveolar ridge following the extraction of anterior maxillary teeth (18).
Regarding the use of ADM in oral cavity reconstruction, Izumi et al (19) developed a human oral mucosa equivalent that was produced ex vivo in a serum-free culture system without a feeder layer. It comprised a stratified layer of autogeneous human oral keratinocytes seeded on top of ADM and was demonstrated to promote wound healing more favorably than AlloDerm alone for human intraoral grafting procedures. In addition, Girod et al (7) compared the efficacy of ADM and split thickness skin grafts in oral cavity reconstruction and concluded that the use of ADM had several advantages over split thickness skin grafts. These included a lack of donor site morbidity, a natural-appearing mucosal surface and lower surgical fees. However, to the best of our knowledge, few studies have been performed in animals to observe the dynamic progress of tissue growth or investigate alterations in molecular biology during reconstruction of intraoral defects over time.

In the present study, observation of the gross appearance between the ADM and control groups indicated that ADM improved the healing rate of oral mucosal wounds and shortened the recovery time while inducing only a low inflammatory response compared with the tissue reaction in an untreated wound. These results are consistent with a previous clinical study (19). Notably, the histological analysis executed by Xiong et al (20) on a palatal mucosa equivalent consisting of cultured oral keratinocytes and porcine ADM indicated that keratinocytes infiltrated the empty spaces of the porcine 
ADM and formed an anchor-like structure, resembling the rete ridges of the native palate mucosa. Thus, it was hypothesized in the current study that ADM serves as a cellular scaffold in oral mucosal defect recovery and guides tissue regeneration. However, the pathological regression of ADM used in surgery, including absorption, degradation or incorporation as an autologous structure into the palate, remains unclear. In a previous study conducted by Ophof et al (21), it was also unclear whether ADM was rapidly degraded or sequestrated. A low level of phagocytic cells was observed in the ADM group compared with the control group, indicating minimal rejection, which may be due to the histocompatibility of ADM (22). Therefore, the likelihood of absorption and degradation of the ADM was decreased. No clear differences were identified between the two groups in gross appearance and pathological features at 4 weeks following surgery, suggesting that ADM had no notable short-term side effects on recovery.

It has previously been demonstrated that ADM may be used as an interpositional connective tissue graft for the repair of mucosal defects (23). Therefore, in the current study, histology was used to investigate the dynamic tissue response of the palatal mucosa. More specifically, the infiltration of inflammatory cells, epithelialization, revascularization and collagen orientation were assessed. Luczyszyn et al (24) and Izumi et al (19) demonstrated similar results by examining the repair of mucosal defects treated with ADM. In the current study, prominent epithelialization and transformation were observed in the ADM group when assessed at week 2 following surgery. In addition, a decreased inflammatory reaction was observed compared with the control group, which was marked by a small number of inflammatory cells and well-organized tissue in the ADM group. Inflammation is always accompanied by remodeling of the extracellular matrices in this mucosa equivalent, which is essential for the formation of granulation tissue (25). Foldable tissues possess a high capacity for epithelialization in order to preserve local anatomy as well as chewing, phonation and deglutition (26). It has been previously demonstrated that the collagen fibers in a healing wound run in the direction of the contraction forces (27). In the current study, the collagen fibers in the tissue of the control group were more randomly oriented compared with those in the ADM group and the wounds treated with ADM exhibited fewer fibroblasts compared with untreated wounds. Revascularization during the recovery progress is necessary for wound healing (28). In the current study, rapid revascularization was observed in ADM-treated defects within 1 week and a wide range of vascularization was present by 2 weeks following surgery. These results are consistent with results presented by Takami et al (29). The mechanisms underlying the faster revascularization observed with ADM grafts compared with untreated wounds was further explored in the current study. It has previous been demonstrated that VEGF is highly expressed by epidermal keratinocytes and is overexpressed during wound healing (30). In the current study, the expression of VEGF during oral mucosal repair with or without ADM treatment was evaluated. We indicated that there was a higher expression of VEGF in the ADM-treated group compared with the control at 2 and 4 weeks following surgery, suggesting that VEGF secreted by an ADM graft may assist vascularization following grafting, which is critical to subsequent graft survival (31). These results provide an explanation for the rapid revascularization present in the ADM-treated group in the present study.

During wound healing, proliferative cell activity is another key component required for the promotion of epithelialization. High GLUT1 expression has previously been reported in the wound margin, with re-epithelialization demonstrated to occur in GLUT1 positive sites (32). This suggests that GLUT1 expression is an indicator of proliferative cell activity during wound healing. It was thus hypothesized that GLUT1 levels are enhanced following wounding, which serves a critical role in the healing of oral wounds. In the current study, it was observed that GLUT1 protein was highly expressed in ADM grafts compared with untreated wounds. However, the use of ADM had no effect on the expression of GLUT1 mRNA. GLUT1 is a membrane protein and member of the glucose transporter family, which acts as a carrier for glucose uptake into cells (33). Kuroki et al (34) identified that GLUT1 expression was present throughout all epithelial cell layers in the oral mucosa and was decreased in parallel with the differentiation gradient. Activation of GLUT1 requires an increased level of glucose (35). The cellular scaffold role of ADM may reduce the consumption of energy so that the cellular level of glucose increases. Under these circumstances, GLUT1 protein levels would subsequently be increased. Therefore, the current study provides cytobiological evidence to indicate that ADM promotes epithelialization and revascularization during wound healing without rejection.

However, the current study had the following limitations: It was performed on individual animals and individual heterogeneity was not considered; the only histological analyses performed used H\&E staining, while the use of more specific stains may have contributed additional information. Furthermore, immunohistochemical analyses to explore the distribution of VEGF and GLUT1 should be performed in future studies.

In conclusion, in comparison with untreated wounds, it appears that palatal wounds treated with ADM stimulate a mild inflammatory response, exhibit rapid epithelialization and revascularization and are accompanied by an increase in VEGF and GLUT1, all leading to the promotion of tissue growth in defect sites. The effects on molecular biology and the histological process of repair for defects of the oral mucosa that have been treated with ADM were elucidated. The results provide evidence supporting the clinical use of ADM, which may lead to improvements in intraoral mucosal repair in humans in the future.

\section{References}

1. Kinikoglu B, Damour O and Hasirci V: Tissue engineering of oral mucosa: A shared concept with skin. J Artif Organs 18: 8-19, 2015.

2. MacNeil S: Progress and opportunities for tissue-engineered skin. Nature 445: 874-880, 2007.

3. Ueda M, Tohnai I and Nakai H: Tissue engineering research in oral implant surgery. Artif Organs 25: 164-171, 2001.

4. Liu J, Bian Z, Kuijpers-Jagtman AM and Von den Hoff JW: Skin and oral mucosa equivalents: Construction and performance. Orthod Craniofac Res 13: 11-20, 2010.

5. Mohd Nor NH, Berahim Z, Ahmad A and Kannan TP: Properties of cell sources in tissue-engineered three-dimensional oral mucosa model: A review. Curr Stem Cell Res Ther 12: 52-60, 2017. 
6. Berthiaume F, Maguire TJ and Yarmush ML: Tissue engineering and regenerative medicine: History, progress, and challenges. Annu Rev Chem Biomol Eng 2: 403-430, 2011.

7. Girod DA, Sykes K, Jorgensen J, Tawfik O and Tsue T: Acellular dermis compared to skin grafts in oral cavity reconstruction. Laryngoscope 119: 2141-2149, 2009.

8. Livesey SA, Herndon DN, Hollyoak MA, Atkinson YH and Nag A: Transplanted acellular allograft dermal matrix. Potential as a template for the reconstruction of viable dermis. Transplantation 60: 1-9, 1995.

9. Rhee PH, Friedman CD, Ridge JA and Kusiak J: The use of processed allograft dermal matrix for intraoral resurfacing: An alternative to split-thickness skin grafts. Arch Otolaryngol Head Neck Surg 124: 1201-1204, 1998.

10. Fischer AH, Jacobson KA, Rose J and Zeller R: Hematoxylin and eosin staining of tissue and cell sections. CSH Protoc 2008: pdb. prot4986, 2008.

11. Livak KJ and Schmittgen TD: Analysis of relative gene expression data using real-time quantitative PCR and the 2(-Delta Delta C(T)) method. Methods 25: 402-408, 2001

12. Wainwright DJ: Use of an acellular allograft dermal matrix (AlloDerm) in the management of full-thickness burns. Burns 21 243-248, 1995.

13. Wong AK, Schonmeyr B, Singh P, Carlson DL, Li S and Mehrara BJ: Histologic analysis of angiogenesis and lymphangiogenesis in acellular human dermis. Plast Reconstr Surg 121: $1144-1152,2008$

14. Buinewicz B and Rosen B: Acellular cadaveric dermis (AlloDerm): A new alternative for abdominal hernia repair. Ann Plast Surg 52: 188-194, 2004.

15. Becker S, Saint-Cyr M, Wong C, Dauwe P, Nagarkar P, Thornton JF and Peng Y: AlloDerm versus DermaMatrix in immediate expander-based breast reconstruction: A preliminary comparison of complication profiles and material compliance. Plast Reconstr Surg 123: 1-6; discussion 107-108, 2009.

16. Adetayo OA, Salcedo SE, Bahjri K and Gupta SC: A meta-analysis of outcomes using acellular dermal matrix in breast and abdominal wall reconstructions: Event rates and risk factors predictive of complications. Ann Plast Surg 77: e31-e38, 2016.

17. Luczyszyn SM, Papalexiou V, Novaes AB Jr, Grisi MF, Souza SL and Taba M Jr: Acellular dermal matrix and hydroxyapatite in prevention of ridge deformities after tooth extraction. Implant Dent 14: 176-184, 2005

18. Fernandes PG, Novaes AB Jr de Queiroz AC, de Souza SL, Taba M Jr, Palioto DB and Grisi MF: Ridge preservation with acellular dermal matrix and anorganic bone matrix cell-binding peptide P-15 after tooth extraction in humans. J Periodontol 82 72-79, 2011

19. Izumi K, Feinberg SE, Iida A and Yoshizawa M: Intraoral grafting of an ex vivo produced oral mucosa equivalent: A preliminary report. Int J Oral Maxillofac Surg 32: 188-197, 2003.

20. Xiong $X$, Zhao Y, Zhang W, Xie W and He S: In vitro engineering of a palatal mucosa equivalent with acellular porcine dermal matrix. J Biomed Mater Res A 86: 544-551, 2008.

21. Ophof R, Maltha JC, Von den Hoff JW and Kuijpers-Jagtman AM: Histologic evaluation of skin-derived and collagen-based substrates implanted in palatal wounds. Wound Repair Regen 12: $528-538,2004$
22. Wainwright D, Madden M, Luterman A, Hunt J, Monafo W, Heimbach D, Kagan R, Sittig K, Dimick A and Herndon D: Clinical evaluation of an acellular allograft dermal matrix in full-thickness burns. J Burn Care Rehabil 17: 124-136, 1996.

23. Kridel RW, Foda H and Lunde KC: Septal perforation repair with acellular human dermal allograft. Arch Otolaryngol Head Neck Surg 124: 73-78, 1998.

24. Luczyszyn SM, Grisi MF, Novaes AB Jr, Palioto DB, Souza SL and Taba M Jr: Histologic analysis of the acellular dermal matrix graft incorporation process: A pilot study in dogs. Int J Periodontics Restorative Dent 27: 341-347, 2007.

25. Yannas IV, Lee E, Orgill DP, Skrabut EM and Murphy GF: Synthesis and characterization of a model extracellular matrix that induces partial regeneration of adult mammalian skin. Proc Natl Acad Sci USA 86: 933-937, 1989.

26. Uğurlu K, Hüthüt I, Ozçelik D, Ozer K, Sakiz D, Yildiz K and Bas L: Epithelialization process of free fascial flaps used in reconstruction of oral cavity mucosa defects in dogs. Plast Reconstr Surg 113: 915-926, 2004

27. Wei PC, Laurell L, Lingen MW and Geivelis M: Acellular dermal matrix allografts to achieve increased attached gingiva. Part 2. A histological comparative study. J Periodontol 73: 257-265, 2002

28. Chaplin JM, Costantino PD, Wolpoe ME, Bederson JB, Griffey ES and Zhang WX: Use of an acellular dermal allograft for dural replacement: An experimental study. Neurosurgery 45: 320-327, 1999.

29. Takami Y, Matsuda T, Yoshitake M, Hanumadass M and Walter RJ: Dispase/detergent treated dermal matrix as a dermal substitute. Burns 22: 182-190, 1996.

30. Szpaderska AM, Walsh CG, Steinberg MJ and DiPietro LA: Distinct patterns of angiogenesis in oral and skin wounds. J Dent Res 84: 309-314, 2005

31. Nakanishi Y, Izumi K, Yoshizawa M, Saito C, Kawano Y and Maeda T: The expression and production of vascular endothelial growth factor in oral mucosa equivalents. Int J Oral Maxillofac Surg 36: 928-933, 2007

32. Lenoir MC, Bernard BA, Pautrat G, Darmon M and Shroot B: Outer root sheath cells of human hair follicle are able to regenerate a fully differentiated epidermis in vitro. Dev Biol 130: 610-620, 1988 .

33. Takahashi H, Kaminski AE and Zieske JD: Glucose transporter 1 expression is enhanced during corneal epithelial wound repair. Exp Eye Res 63: 649-659, 1996.

34. Kuroki S, Yokoo S, Terashi H, Hasegawa M and Komori T: Epithelialization in oral mucous wound healing in terms of energy metabolism. Kobe J Med Sci 55: E5-E15, 2009.

35. Liemburg-Apers DC, Wagenaars JA, Smeitink JA, Willems PH and Koopman WJ: Acute stimulation of glucose influx upon mitoenergetic dysfunction requires LKB1, AMPK, Sirt2 and mTOR-RAPTOR. J Cell Sci 129: 4411-4423, 2016.

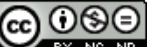

This work is licensed under a Creative Commons Attribution-NonCommercial-NoDerivatives 4.0 International (CC BY-NC-ND 4.0) License. 